\title{
Editorial
}

\section{THE FAILURE OF GEOMETRY: TIME FOR GEARS!}

A schematic representation of the manufacture of goods and management of associated wastes frequently results in an extensive use of geometrical figures: lines, triangles and circles.

The task of these geometrical figures is to portray a graphic synthesis of concepts and strategies. Therefore, the actual concept underlying the basis of the representation is of fundamental importance. The concept first and foremost, followed by the figure that should not overpower the concept by straining, conditioning or, worse still, distorting it.

As an example, the concept of "take-make-waste" based on the use of a renewable or non-renewable resource in the manufacture of goods and subsequent disposal of associated wastes represents the so-called linear approach depicted by a straight line. A line starts and ends at fixed points. The simplest linear system in product and waste management (Figure 1A) moves directly from the soil (resource extraction) and ends up in the soil (waste disposal). A line has no branches, does not retrace its steps, does not reflect and has no afterthoughts. Important material resources are buried in landfills. No form of consideration for the safeguard of non-renewable resources, environmental protection is of a passive nature and environmental sustainability is virtually completely overlooked. Indeed, proces- ses such as the recovery of biogas from landfills or energy recovery following waste incineration deviate from the perfect linearity of the system.

As the concepts of a hierarchy and integrated waste management took hold (initially established in Europe by EU Directive 75/442/EEC ), the line was gradually replaced by a top-down triangle listing the different stages of waste management in a top-down priority order (Figure 1B). This representation indicates a priority order of Prevention, Preparing for re-use, Recycling, Energy recovery and Disposal based on increasingly minimised quantities of waste. Landfills are viewed as a bin into which the smallest possible quantities of waste should be introduced, thus resulting in a lower risk of pollution and occupation of space.

The rigidity of the triangular geometric form elicits an idealistic order which is scarcely relatable to the real world. For example, nowhere in the world are wastes subjected prevalently to prevention measures, and little is undertaken at a regulatory level to promote and apply these measures. The triangles are steeped in moralism. Landfills should be in a position to become virtually obsolete, but people simply seem to be unable to manage without them (Cossu et al., 2020). The triangles moreover seem to convey dreams rather than the actual situation. Furthermore, the rigidity of

\section{\begin{tabular}{l|l|l|l|l|} 
RESOURCE EXTRACTION & PRODUCTION & DISTRIBUTION & CONSUMPTION & WASTE DISPOSAL
\end{tabular}}

\section{A}
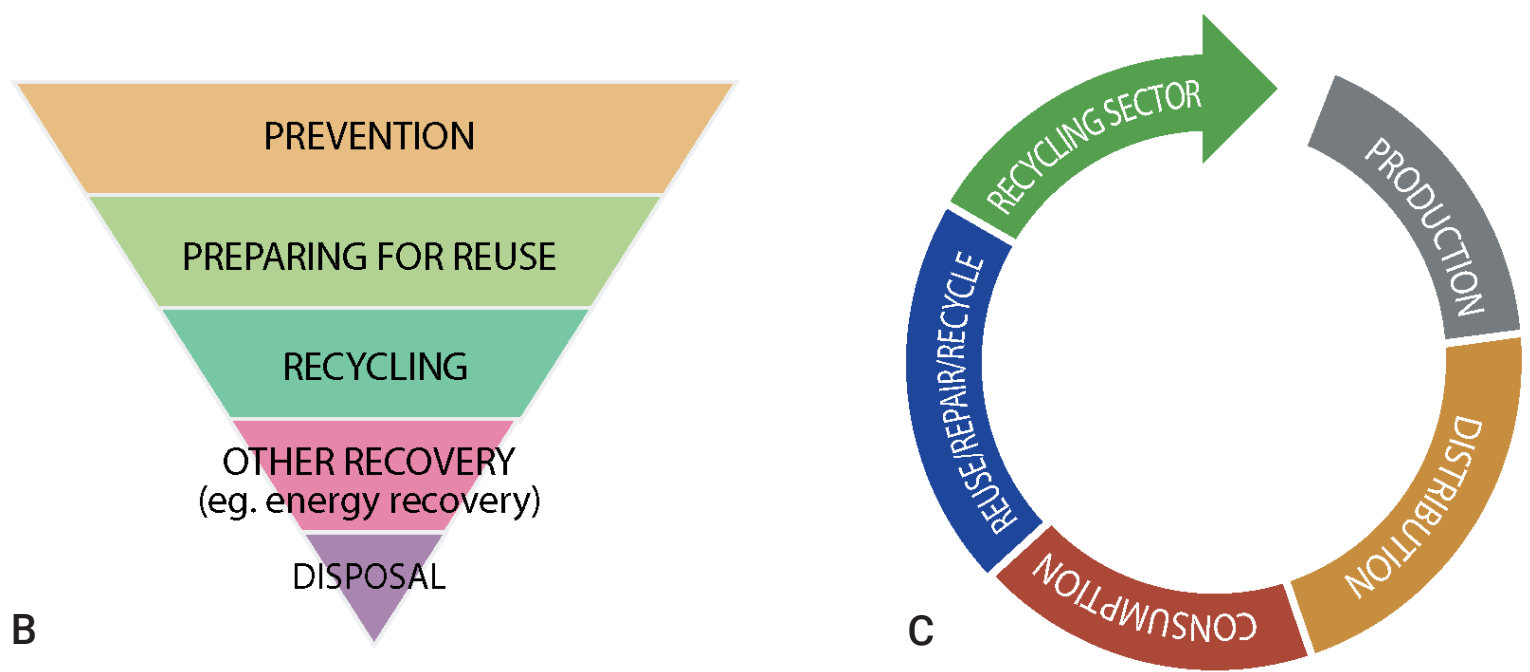

FIGURE 1: Schematic representation of a series of concepts relating to the production of goods and management of associated wastes.

Detritus / Volume 13 - 2020 / pages 1-2

https://doi.org/10.31025/2611-4135/2020.14034

(c) 2020 Cisa Publisher. Open access article under CC BY-NC-ND license 


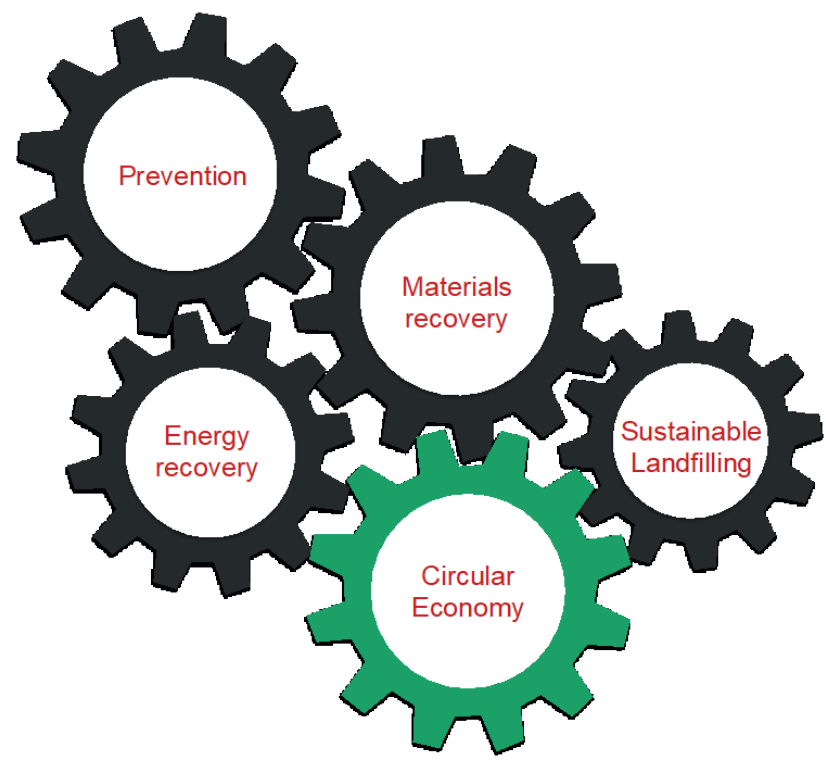

FIGURE 2: Representation of a balanced solid waste management strategy aimed at achieving the functional (environmental, technical and economic) management of Circular Economy.

the triangle makes us lose sight of the virtuous role played by landfill in closing the material loop. Within triangles, materials seem almost to volatise, to disappear!

To now proceed to the circle. In all cultures, the circle has long conveyed the notion of perfection and movement. In waste management, the circle is inevitably elevated to the symbol of Circular Economy, i.e. a system rotating around the prevention, reuse, recovery and recycling of wastes, stoking various production cycles with secondary raw materials. On a European level, the aim declared was to "close the loop" of product lifecycles bringing benefits for both the environment and the economy. The circle is well aware of and satisfies the need to contrast the depletion of non-renewable resources through the reuse of resources present in wastes, thus highlighting the benefits to be gained in reducing waste volumes forwarded to landfill and raising awareness amongst those who generate the wastes with an aim to improving waste quality and reducing waste quantities. However, this geometrical image of the perfect circle creates dangerous expectations and boosts the myth of the Zero Waste principle, seen idealistically as a waste management system no longer reliant on landfills or thermal waste treatment plants. In particular, the perfect circle fails to take into account the following fundamental aspects:

- not all materials are recyclable, and those that are cannot be recycled ad infinitum;

- hazardous and persistent chemical substances present in the products forwarded to recycling tend to accumulate in the recycled materials and residues;
- the material cycle should necessarily be closed in line with the principle of Back to Earth to control the global diffusion of contaminants; accordingly, if what is taken from the land is not returned to the land in an uncontaminated form, it will linger perilously in the environment (Grossule, 2020);

- in closing the material loop, the strategically important role played by landfill is not taken into account;

- the entire waste management system is intended to be sustainable and exhaust all negative environmental impacts within the time frame of one generation; this aspect however does not take into account landfills, the regulations for which are not geared towards environmental sustainability nor the technical specifications suited to achieving the same;

- the issue of climate change is viewed prevalently in terms of minimisation of greenhouse gas emissions, neglecting the sequestering and immobilisation of carbon ("carbon sink") which, once again, could be achieved by landfills.

Following these failures of the perfect geometric forms, perhaps the time has come to turn to other forms of graphic representation that indulge less on rhetoric and demagoguery and are firmly anchored to the principles regulating the exchange of energy and materials in the natural cycles in which all stages play a role, in which there are no good or bad solutions, where everything is harmoniously correlated.

The natural cycles do not seek moralistic perfection but tend towards the rationality of nature. Where everything moves in line with the principle of mechanical gears, having instrumental, interconnected roles perpetuated throughout the diverse processes, transformations and reserves of resources, products and wastes.

Explicitly, the representation of product and waste management as a system of gears in which each piece is fundamental clearly illustrates how prevention, material recovery, energy recovery, and sustainable landfill are all of priority importance in guaranteeing an equilibrium in the functioning of Circular Economy.

This is a message of paramount importance to be conveyed to people in order to avoid preconceived oppositions to thermal treatment plants and sustainable landfills.

Raffaello Cossu

University of Padova, Italy

raffaello.cossu@gmail.com

\section{REFERENCES}

Cossu R., Grossule V., Lavagnolo M.C. (2020). What about residues from Circular Economy and role of landfilling. Detritus, vol. 9, 1-3, https://doi.org/10.31025/2611-4135/2020.13920

Grossule V. (2020). Final quality of a sustainable landfill and postclosure management. Detritus, vol. 13, 148-159, https://doi. org/10.31025/2611-4135/2020.13999 